\title{
Effect of optical waveguiding mechanism on the lasing action of chirped InAs/AIGaInAs/InP quantum dash lasers
}

\author{
M. Z. M. Khan ${ }^{\mathrm{a}}$, Tien K. Ng ${ }^{\mathrm{a}}$, C. -S. Lee ${ }^{\mathrm{b}}$, P. Bhattacharya ${ }^{\mathrm{b}}$, and Boon S. Ooi*a \\ ${ }^{a}$ Photonics Laboratory, King Abdullah University of Science \& Technology (KAUST), Thuwal \\ 23955-6900, Kingdom of Saudi Arabia (KSA) \\ ${ }^{\mathrm{b}}$ Department of Electrical Engineering and Computer Science, University of Michigan, 1301, Beal \\ Avenue, Ann Arbor, Michigan 48109-2122, United States of America (USA) \\ *corresponding author: boon.ooi@kaust.edu.sa
}

\begin{abstract}
We report on the atypical emission dynamics of InAs/AlGaInAs/InP quantum dash (Qdash) lasers employing varying AlGaInAs barrier thickness (multilayer-chirped structure). The analysis is carried out via fabry-perot (FP) ridge (RW) and stripe waveguide (SW) laser characterization corresponding to the index and gain guided waveguiding mechanisms, respectively, and at different current pulse width operations. The laser emissions are found to emerge from the size dispersion of the Qdash ensembles across the four Qdash-barrier stacks, and governed by their overlapping quasi-zero dimensional density of states (DOS). The spectral characteristics demonstrated prominent dependence on the waveguiding mechanism at quasi-continuous wave (QCW) operation (long pulse width). The RW geometry showed unusual spectral split in the emission spectra on increasing current injection while the SW geometry showed typical broadening of lasing spectra. These effects were attributed to the highly inhomogeneous active region, the nonequilibrium carrier distribution and the energy exchange between Qdash groups across the Qdash-barrier stacks. Furthermore, QCW operation showed a progressive red shift of emission spectra with injection current, resulted from active region heating and carrier depopulation, which was observed to be minimal in the short pulse width (SPW) operation. Our investigation sheds light on the device physics of chirped Qdash laser structure and provides guidelines for further optimization in obtaining broad-gain laser diodes.
\end{abstract}

Keywords: Quantum dash lasers, ridge-waveguide laser, broad-area lasers, quasi-continuous wave operation.

\section{INTRODUCTION}

The InAs/GaAs and InAs/InP based material technology have attracted both scientific attention and technological deployment since the utilization of quantum confined nanostructures as the active regions have provided substantial improvement in the device characteristics apart from long wavelength emissions [1], [2]. In particular, the laser diode (LD) based on InAs/InP quantum-dot (Qdot)/Qdash active region has shown impressive progress due to their superior device performance, such as low threshold current density, high differential gain, etc. [2], [3]. The advancement in Qdot/Qdash epitaxy growth techniques, and the associated numerical models [3], [4] has contributed to these achievements. Moreover, the recently reported broadband emission from Qdash lasers spanning $\sim 41 \mathrm{~nm}(-3 \mathrm{~dB}$ bandwidth) at relatively high emission power in C- and L-bands further strengthened their possible application in optical long haul communication [5]. Laser diodes with high beam quality and stable power, such as RW LD, are generally utilized for these applications. However, the reported broadband lasers utilized SW geometry. Besides, spectral modulations due to lateral cavity resonances, and leaky mode resonances [6] have been reported from InAs/GaAs RW lasers, which are undesirable. Therefore, it becomes important to investigate the RW LDs on InP substrate platform for such unusual emission dynamics.

In the paper, we propose to utilize a chirp laser structure to compare the lasing dynamics of RW and SW LDs at short pulse width (SPW) and quasi continuous wave (QCW) operations. Our results showed unusual lasing characteristics from the RW LDs which we attributed to various mechanisms describe in the following.

Novel In-Plane Semiconductor Lasers XII, edited by Alexey A. Belyanin, Peter M. Smowton,

Proc. of SPIE Vol. 8640, 864005 - @ 2013 SPIE · CCC code: 0277-786X/13/\$18 · doi: 10.1117/12.2003462 
(a)

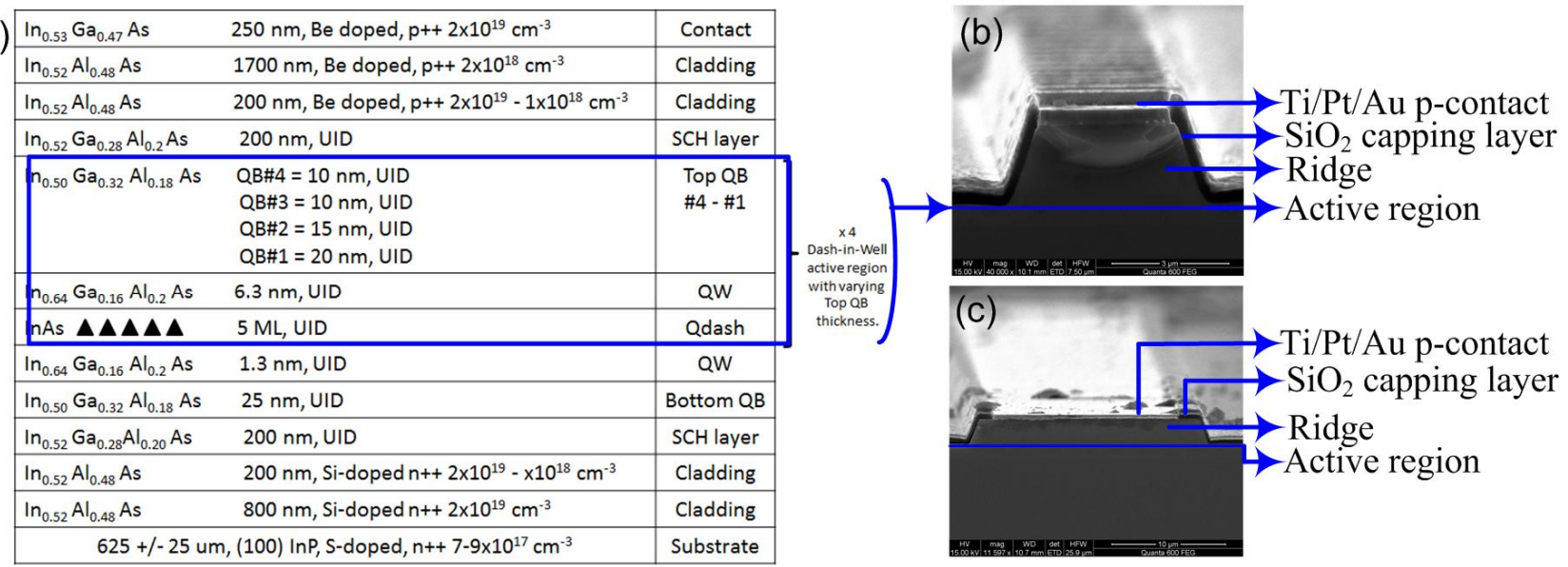

Fig. 1 (a) Schematic of InAs/InP Qdash laser diode with a chirp structure, and Scanning electron microscopy (SEM ) images of fabricated RW (b) and SW (c) devices at magnifications of 40,000 $\times$ and $11600 \times$, respectively.

\section{LASER STRUCTURES}

The self-organized InAs/InP Qdash device chirp structure was grown by molecular beam epitaxy on (100) oriented, Sdoped n-type InP substrate as shown in Fig. 1(a). The LD is in p-i-n configuration with a $200 \mathrm{~nm}$ thick $\operatorname{In}_{0.52} \mathrm{Ga}_{0.28} \mathrm{Al}_{0.2} \mathrm{As}$ undoped separate confinement heterostructure $(\mathrm{SCH})$ layer lattice matched to InP substrate. The lower cladding consists of $200 \mathrm{~nm}$ thick $\operatorname{In}_{0.52} \mathrm{Al}_{0.48} \mathrm{As}$ Si-doped $\left(2 \times 10^{18} \mathrm{~cm}^{-3}\right)$ layer. The $1900 \mathrm{~nm}$ thick $\operatorname{In}_{0.52} \mathrm{Al}_{0.48} \mathrm{As}$ upper cladding and 250 $\mathrm{nm}$ thick $\mathrm{In}_{0.53} \mathrm{Ga}_{0.47} \mathrm{As}$ contact layers are and Be-doped at concentrations of $2 \times 10^{18} \mathrm{~cm}^{-3}$ and $2 \times 10^{19} \mathrm{~cm}^{-3}$, respectively. The waveguide core centre comprises of undoped quantum-dash-in-asymmetric-well active region with 4 alternating stacks of $1.3 \mathrm{~nm}$ thick compressively strained $\mathrm{In}_{0.64} \mathrm{Ga}_{0.16} \mathrm{Al}_{0.2} \mathrm{As}$ well layer, a $5 \mathrm{ML}$ thick InAs dash layer, and a $6.3 \mathrm{~nm}$ thick compressively strained $\mathrm{In}_{0.64} \mathrm{Ga}_{0.16} \mathrm{Al}_{0.2} \mathrm{As}$ well layer. Each quantum-dash-in-asymmetrical-well stack is separated by varying thickness $\left(10 \mathrm{~nm}, 10 \mathrm{~nm}, 15 \mathrm{mn}\right.$, and $20 \mathrm{~nm}$ ) tensile-strained $\operatorname{In}_{0.50} \mathrm{Ga}_{0.32} \mathrm{Al}_{0.18} \mathrm{As}$ top barriers, on a single $\mathrm{In}_{0.50} \mathrm{Ga}_{0.32} \mathrm{Al}_{0.18} \mathrm{As} 25 \mathrm{~nm}$ thick lower barrier. More details of the structure are shown in Fig. 1(a). RW (width of 2-4 $\mu \mathrm{m}$ and length of 450-2000 $\mu \mathrm{m}$ ) and SW (width of 15-50 $\mu \mathrm{m}$ and length of 450-2000 $\mu \mathrm{m}$ ) LDs were fabricated and cleaved, employing standard techniques with uncoated facets. The scanning transmission microscopy (SEM) of both types of laser diodes geometry fabricated for the current work are shown in Figs. 1(b) and (c). The ridge height is fixed at $2 \mu \mathrm{m}$ and the LDs were tested at room temperature at different input pulse widths; $0.5 \mu \mathrm{s}$ (SPW), $2 \mu \mathrm{s}$, and $100 \mu \mathrm{s}$ (QCW).

\section{RESULTS AND DISCUSSION}

\subsection{Material Characterization}

The chirped InGaAlAs barrier layer thickness in the InAs/InP Qdash laser structure was first evaluated utilizing photoluminescence (PL) spectroscopy at $77 \mathrm{~K}$ using $1064 \mathrm{~nm}$ diode pumped solid-state (DPSS) laser as excitation source. The results are shown in Fig. 2. At $1.5 \mathrm{~W} / \mathrm{cm}^{2}$ the emission peak was observed at $\sim 1540 \mathrm{~nm}$ and gradually blue shifted with increasing excitation power density, with broadening of the PL spectra. At high excitation of $3000 \mathrm{~W} / \mathrm{cm}^{2}$ the peak emission wavelength is $\sim 1462 \mathrm{~nm}$ due to band-filling. In addition, another shoulder developed at the short wavelength side of the PL spectra with peak wavelength $\sim 1414 \mathrm{~nm}$. The PL full-width at half-maximum (FWHM) at $3000 \mathrm{~W} / \mathrm{cm}^{2}$ is $\sim 151 \mathrm{~nm}$ which implies highly inhomogeneous active region as a result of intentional chirping of barrier layer thickness which affect the vertical strain and influencing the inhomogeneity of the subsequent Qdash layer The emission peaks at $\sim 1540 \mathrm{~nm}$ and $\sim 1462 \mathrm{~nm}$ were attributed to the Qdash layers with $10 \mathrm{~nm}$ barrier layers having larger average dash height $\left(Q_{-} L\right)$. The formation of emission shoulder in the $3000 \mathrm{~W} / \mathrm{cm}^{2} \mathrm{PL}$ spectra suggested the emission from $15 \mathrm{~nm}$ barrier Qdash layer with intermediate average height dashes $\left(Q_{-} M\right)$. The emission hump from the thickest- 
barrier dash layer $(20 \mathrm{~nm})$ cannot be observed clearly. However, deconvolving the spectra into four Gaussian curves gives the emission peak of $20 \mathrm{~nm}$ barrier Qdash layer at $\sim 1380 \mathrm{~nm}$, corresponding to the short average height dashes $\left(Q \_S\right)$. The ultra broad PL FWHM from this structure is promising for realizing broadband sources. Our studies further show that carriers are non-uniformly distributed among inhomogeneously broadened dash ensembles, particularly due to the dash heights and shape, as depicted in the atomic force microscopy (AFM) image (see inset of Fig. 2), resulting in a broad emission by the collective contribution from these dashes. We also performed transmission electron microscopy (TEM) across the [110] and [1-10] directions, on the laser structure. The measurements revealed unequal height variation across the Qdash stacks with the average height of the $10 \mathrm{~nm}$ barrier Qdash stack having the largest value, thus confirming the varying dash height across the Qdash stacks.

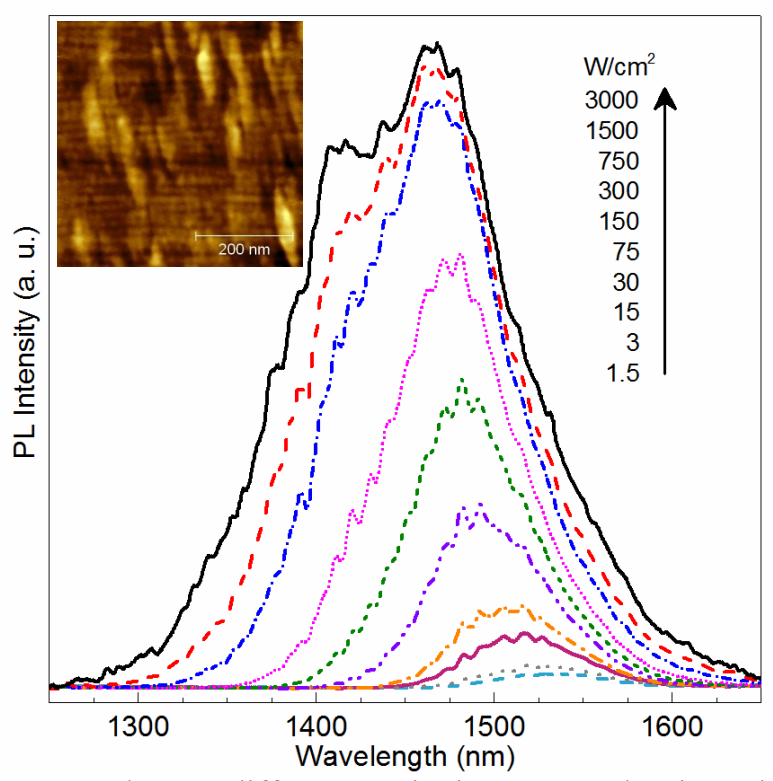

Fig. 2. The $77 \mathrm{~K}$ photoluminescence taken at different excitation power density. The inset showed the atomic force microscopy (AFM) image of Qdashes.

\subsection{Device Characterization}

Fig. 3(a) shows the $L-I$ characteristics of the $2 \times 1000 \mu \mathrm{m}^{2}$ Qdash RW LD at different pulse widths. The progressive decrease in the output power (per facet) with increase pulse width is noticeable, which is an obvious indication of device heating. Moreover, kinks are observed in the $L-I$ curve which are observed to be sharp in the QCW injection, and not so during SPW operation. This is attributed to the competing lasing actions from different confined energy levels due to the occurrence of energy exchange between short and long wavelength lasing modes [7], as can be seen in the lasing spectras depicted in Figs. 3(b) and 3(c). In addition, this can also be a result of excessive photon re-absorption in the system, particularly in the QCW mode, as a result of device heating which led to carrier deficiency in the active region (due to carrier depopulation). The behavior of RW LD at these two operating modes is observed to be different in terms of emission spectra. A spectral split at high injection $\left(\sim 3.3 I_{t h}\right)$ was observed in the QCW mode (Fig. 3(c)) resulting in the formation of kink in the $L-I$ curve around the same injection values. On the other hand, the SPW did not show spectral splitting (Fig. 3(b)), and instead a single emission lobe is observed. However, a sudden substantial broadening of the spectra around the current injection was observed, coinciding with the observation of a weak kink in the $L-I$ curve. This suggested that SPW mode of operation constitute a carrier sufficient system in which device heating is minimal since negligible red shift in the central lasing wavelength with increasing current injection was observed. The broadening of the spectra was a result of collective lasing from different Qdash ensembles $\left(Q_{-} L, Q_{-} M\right.$, and $\left.Q_{-} S\right)$ concurrently. However, in the case of QCW mode, the split forms a group of short wavelength longitudinal modes (due to $Q \_S$ ), which progressively blue shifted with diminishing intensity, and the long wavelength modes (due to $Q_{-} L$ ) red shifted, thus forming a negligible intensity group of intermediate wavelength modes $\left(Q_{-} M\right)$, leading to a gap in the lasing spectra. We attributed this observation to the large photon re-absorption among the highly inhomogeneous active region with increasing current injection where in the $Q_{-} M$ behave as carrier feeders to $Q_{-} L$ (which possesses larger modal gain and 
higher DOS). Concurrently, the $Q \_S$ also fed $Q \_M$ but this mechanism was moderate because of the lower DOS and smaller modal gain of $Q_{-} M$ dashes. Hence, the shorter height dash groups are able to sustain lasing apart from feeding the intermediate height dash ensembles. This might be the reason that the shorter height dash groups sustained lasing apart from feeding the intermediate height dash ensembles. It was noted that the long wavelength dash group started blue shifting, and broadening once the short wavelength group disappeared which happened within the span of $\sim 1.0 I_{t h}$ increase in current injection. This might be due to band filling effect where the carriers recombine through the higher subbands of dashes resulting in short wavelength stimulated emission, which in turn accelerates the carrier feeding mechanism from $Q_{-} S$ dashes, thus reducing the lasing intensity of these dash groups. The trend continues until $\sim 5.0 I_{t h}$ where the spectral split is again observed suggesting a periodic behavior. Since the above phenomenon happen during QCW operation wherein various non-linear effects (for instance, spectral hole burning) comes into picture, this observation might also be regarded as a collective observation from these effects in addition to the active region inhomogeneity and device heating. Such a behavior, to our knowledge, has been reported for the first time.
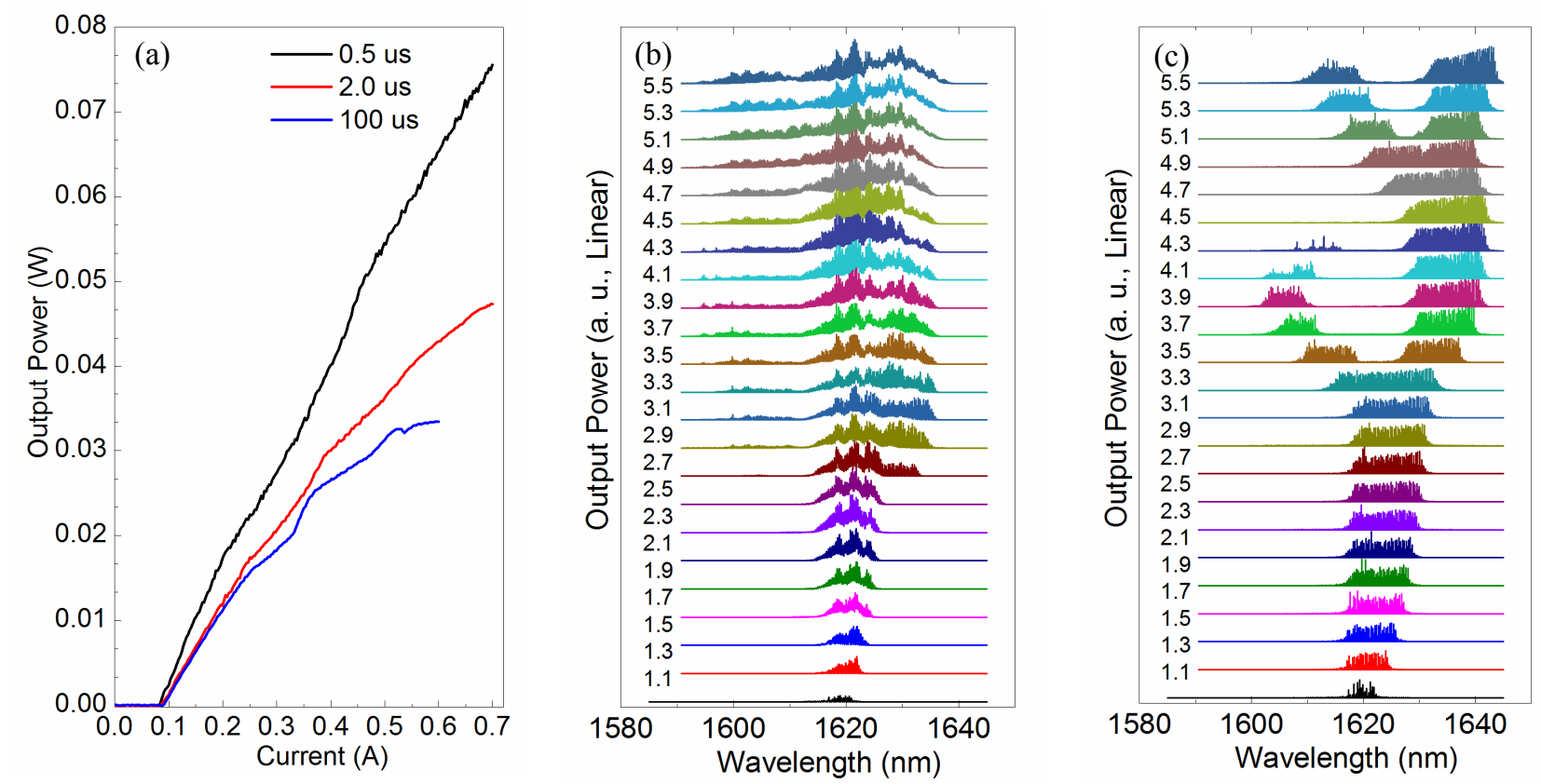

Fig. 3. Room temperature (a) $L-I$ characteristics at different pulse width operation, and the lasing spectra at different current injection for SPW (b) and QCW (c) operation, for the $2 \times 1000 \mu \mathrm{m}^{2} \mathrm{RW} \mathrm{LD}$. The threshold current, $I_{t h}$, is 75 $\mathrm{mA}$, and the spectras were taken at a current step of $0.2 J_{t h}$ above threshold.

Fig. 4(a) shows the $L-I$ curve for the SW Qdash LD at all injection levels, and at different pulse width operations. The corresponding lasing spectra for the SPW and QCW operations are shown in Fig. 4(b) and 4(c). A smooth $L-I$ curve is observed in this particular laser waveguide geometry with no kinks. Moreover, the lasing spectra of both, SPW and QCW injections show only one group of longitudinal modes. Likewise in the RW case, the QCW operation on the SW Qdash laser showed early output power roll-off with reduced slope efficiency, which is again an indication of thermal heating, and thus leading to red shifting of the emission spectra. In this case as well, the spectra of both the operating modes broadened with increasing current injection, however, the lasing wavelength coverage (calculated at $-25 \mathrm{~dB}$ ) was observed to be small compared to the RW geometry results. For instance, the wavelength coverage for the SPW (QCW) mode is $28 \mathrm{~nm}(34 \mathrm{~nm})$ for the current SW LD compared to $46 \mathrm{~nm}(53 \mathrm{~nm})$ for the RW $\mathrm{LD}$, at a fixed current of $4.0 I_{t h}$. This reduced broadening from the SW geometry might be related to the following: (i) device heating since the thermal resistance is a function of active region dimensions [8], which results in substantial device heating in the RW laser geometry, (ii) The loss mechanisms which is again substantial in the RW LD (for instance, scattering losses at the side walls, intense photon re-absorption, etc.), that increases the total device loss and hence $I_{t h}$. Since, these two parameters are inter related, and affects the distribution of carriers in the active region, it is highly probable that these laser waveguide geometries behave differently in the stimulated emission regime, consistent 
with our observations. Moreover, this type of observation from RW and SW LDs under QCW operation is observed throughout all the laser cavity lengths tested. The spectral modulation in the lasing spectra of Fig. 2 and 3 came from the external cavity formation between the laser facet and the coupling fiber facet.
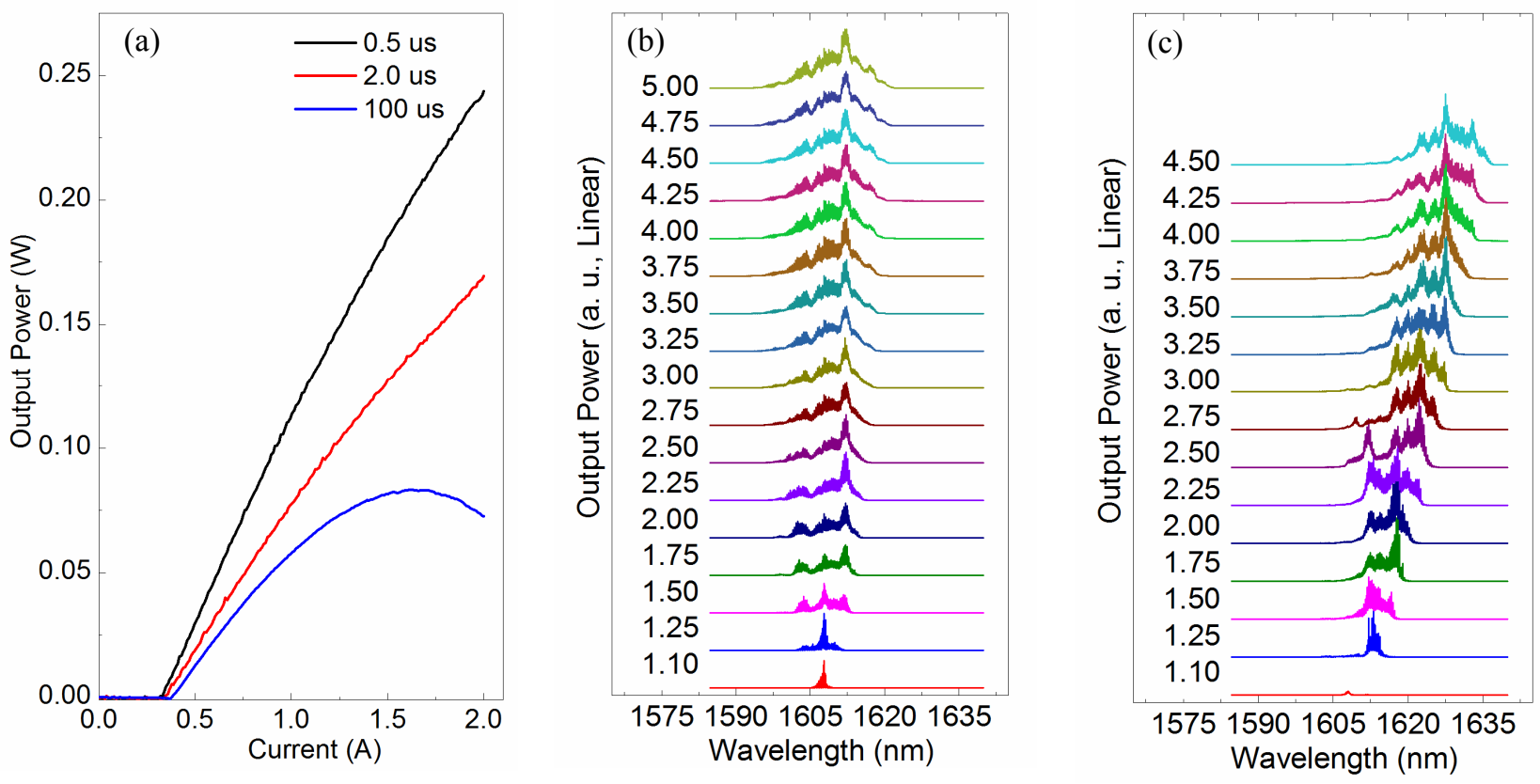

Fig. 3. Room temperature (a) $L-I$ characteristics at varying pulse width operations, and the lasing spectra at different current injection values for the (b) SPW, and (c) QCW operations for the $2 \times 1000 \mu \mathrm{m}^{2} \mathrm{SW}$ LD. The threshold currents were $I_{t h}=0.31 \mathrm{~mA}$, and $0.33 \mathrm{~mA}$ for the SPW and QCW modes, respectively. The spectras were taken at a current step of $0.25 J_{t h}$ above threshold.

\section{CONCLUSION}

In conclusion, we have compared the lasing spectra of RW and SW Qdash lasers with increasing current injection above threshold value, and at two different current pulse width operations. The RW LDs showed unusual spectral split in the lasing emission while the SW LDs show a single emission lobe, under the QCW operation, while a single lasing emission lobe was observed from both the geometries under SPW mode. The results indicated that excessive device heating, various loss mechanisms and non-linear effects, in the laser active region governs the spectral behavior. Our results shed light on the device physics of Qdashes under highly inhomogeneous active region, which is essential in realizing high performance broadband telecommunication devices .

\section{ACKNOWLEDGMENT}

The work was supported by King Abdullah University of Science and Technology’s Competitive Research Grant CRG1-2012-OOI-010.

\section{REFERENCES}

[1] M. Sugawara, N. Hatori, M. Ishida, H. Ebe, Y. Arakawa, T. Akiyama, K. Otsubo, T. Yamamoto and Y. Nakata, "Recent progress in self-assembled quantum-dot optical devices for optical telecommunication: temperature- 
insensitive $10 \mathrm{~Gb} \mathrm{~s}^{-1}$ directly modulated lasers and $40 \mathrm{~Gb} \mathrm{~s}^{-1}$ signal-regenerative amplifiers," J. Phys. D, 38, 21262134 (2005).

[2] F. Lelarge, B. Dagens, J. Renaudier, R. Brenot, A. Accard, F. van Dijk, D. Make, O. Le Gouezigou, J. Provost, and F. Poingt, "Recent advances on InAs/InP quantum dash based semiconductor lasers and optical amplifiers operating at 1.55 m", IEEE J. Sel. Topics Quantum Electron., 13(1), 111-124 (2007).

[3] F. Grillot, K. Veselinov, M. Gioannini, I. Montrosset, J. Even, R. Piron, E. Homeyer and S. Loualiche, "Spectral Analysis of $1.55 \mu \mathrm{m}$ InAs-InP (113)B Quantum-Dot Lasers Based on a Multipopulation Rate Equations Model," IEEE J. Quantum Electron., 45, 872-878 (2009).

[4] M. Z. M. Khan, T. K. Ng, U. Schwingenschlogl, P. Bhattacharya, and Boon S. Ooi, "Modeling the lasing spectra of InAs/InP quantum dash lasers," Appl. Phys. Lett, 98, 101105 (2011).

[5] H. Djie, C. Tan, B. Ooi, J. Hwang, X. Fang, Y. Wu, J. Fastenau, W. Liu, G. Dang and W. Chang, "Ultrabroad stimulated emission from quantum-dash laser", Appl. Phys. Lett, 91, 111116 (2007).

[6] D. Ouyang, R. Heitz, N. Ledentsov, S. Bognar, R. Sellin, C. Ribbat and D. Bimberg, "Lateral-cavity spectral hole burning in quantum-dot lasers," Appl. Phys. Lett, 81, 1546 (2002).

[7] C. Tan, H. Djie, Y. Wang, C. Dimas, V. Hongpinyo, Y. Ding and B. Ooi, "The Influence of Nonequilibrium Distribution on Room-Temperature Lasing Spectra in Quantum-Dash Lasers," IEEE Photon. Technol. Lett., 21, 3032 (2009).

[8] L. A. Coldren, S. W. Corzine, [Diode lasers and photonic integrated circuits], Wiley, New York, USA, (1995). 